\title{
Application Article \\ Construction and Capacity Analysis of High-Rank LoS MIMO Channels in High Speed Railway Scenarios
}

\author{
Jingya Yang, Bo Ai, and Zhangdui Zhong \\ State Key Laboratory of Rail Traffic Control and Safety, Beijing Jiaotong University, Beijing 100044, China \\ Correspondence should be addressed to Bo Ai, aibo@ieee.org
}

Received 22 July 2012; Revised 22 October 2012; Accepted 22 October 2012

Academic Editor: Thomas Kürner

Copyright () 2012 Jingya Yang et al. This is an open access article distributed under the Creative Commons Attribution License, which permits unrestricted use, distribution, and reproduction in any medium, provided the original work is properly cited.

The validity of the maximum capacity criterion applied to realize high-rank line-of-sight (LoS) multiple-input multiple-output (MIMO) channels is investigated for high speed railway scenarios. Performance is evaluated by ergodic capacity. Numerical results demonstrate that by simply adjusting antenna spacing according to the maximum capacity criterion, significant capacity gains are achievable. We find relatively low sensitivity of the system to displacements from the optimal point and angle in relatively short range. Thus, we present two proposals to reconfigure antenna arrays so as to maximize LoS MIMO capacity in the high speed railway scenarios.

\section{Introduction}

MIMO presents an attractive solution for meeting the requirements of next generation wireless communication system for the high speed railway. Since a high speed data rate is required for efficient voice and data transmission services in the future railways, and the bandwidth resources for the railway are limited, the capacity cannot be improved through increasing bandwidth. So MIMO is considered as an effective technique in long-term evolution for railway (LTE-R) to ensure the efficiency and reliability for data transmissions [1].

The development of MIMO systems over the past decade is based on the assumption of i.i.d Rayleigh fading [2]. Although the assumption simplifies the analysis of MIMO systems, its validity is often violated due to either an specular wavefront or a strong direct component existing; then, the entries of the channel matrix can be more effectively modeled by the Ricean distribution. Conceptually, LoS propagation is viewed to limit MIMO advantages because the channel matrix is normally rank deficient $[3,4]$. But some recent investigations have questioned this belief and proposed design methodologies in order to achieve subchannel orthogonally. The orthogonality of subchannels is a key condition for capacity maximization [5-9] and most methodologies are realized by placing the antenna elements sufficiently far apart.

The common characteristics of the above mentioned works are facts about the MIMO performance in a relatively short distance between transmitters and receivers, and meanwhile the low mobility of vehicles in scenarios. Thus, the performance of MIMO optimization design in high speed railways needs to be reassessed under a realistic high speed railway environment. In most scenarios of the high speed railway, the base stations are located less than $30 \mathrm{~m}$ away from the tracks, and most of the BS antenna heights are more than 30 meters, hence there are always a strong line-of-sight (LoS) path between the transmitters and the receivers. Moreover, the channels exhibit a sparse multipath structure due to the lack of sufficient scatters in the railway environment [10]. The channel consists of a direct LoS path and sparse multipath, which is known as LoS MIMO channel. The analysis of small-scale fading characteristics in viaduct scenarios is derived from measurements taken along the "Beijing-Tianjin" high-speed railway of China, refer to [11], which indicates that Ricean $K$-factor increases when the train gets closer to the base station; meanwhile, the number of resolvable paths and RMS spread for delay firstly increase and then decrease. 


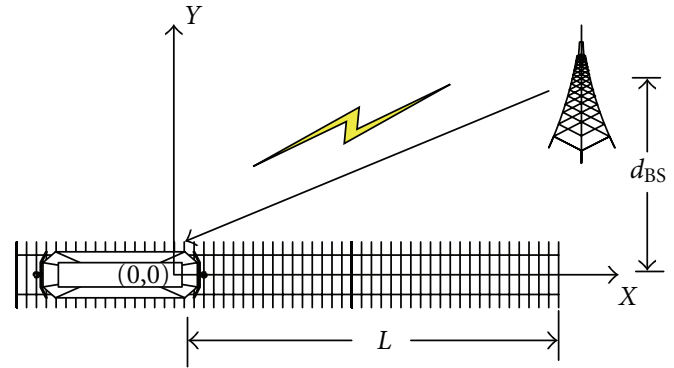

FIgURE 1: The simulation scenario.

In light of these facts, D2a scenario of WINNER II channel model is adopted, which is a realistic high speed railway transmission multipath propagation channel. The primary goal of the present paper is to verify the maximum capacity criterion in the high speed railway and then propose the methodologies for reconfigurable antenna arrays on maximizing the ergodic capacity of MIMO communication links through coverage area of the base station in viaduct scenarios.

The remainder of this paper is organized as follows. Section 2 discusses the simulation scenario and the WINNER II Channel Model. Section 3 develops the maximum capacity criterion. Section 4 concentrates on comparison and analysis of the simulation result and proposes two optimum proposals for reconfiguration antenna array in viaduct scenarios. Finally, Section 5 concludes this paper and summarizes the key findings.

\section{High Speed Railway Channel Model}

2.1. Scenario Description. According to the environment characteristics of high speed railways, D2a scenario is selected. D2a represents radio propagation in environments where MS is moving, possibly at very high speed, in a rural area. The link between the fixed network and the moving network (train) is typically an LoS type.

Figure 1 shows the setting for simulation in high speed railway scenarios. BS is located $14 \mathrm{~m}$ off the track and the height of this transmitter antenna array is $32 \mathrm{~m}$ above the ground. The receiver antenna array is installed on the top of the engine and its height is $3 \mathrm{~m}$ above the ground. The velocity of the train is set to $360 \mathrm{~km} / \mathrm{h}$ towards the BS, and the track is parallel to the $X$-axis. The center frequency and bandwidth are $2.6 \mathrm{GHz}$ and $100 \mathrm{MHz}$, respectively. In Figure 1, the geometry parameters are set to $L=1000 \mathrm{~m}$, $d_{\mathrm{BS}}=14 \mathrm{~m}$, thus BS is located at the centre $(500,14)$.

2.2. WINNER II Channel Model. In WINNER II D2a channel model, each channel realization is generated by summing contributions of eight clusters; each cluster is composed of twenty subpaths, which are associated with different delay, power, angle-of-arrival (AOA), and angle-of-departure (AOD) [12]. As illustrated in Figure 2, consider a single link of a MIMO system with an $S$ elements ULA for BS and a $U$ elements ULA for MS.

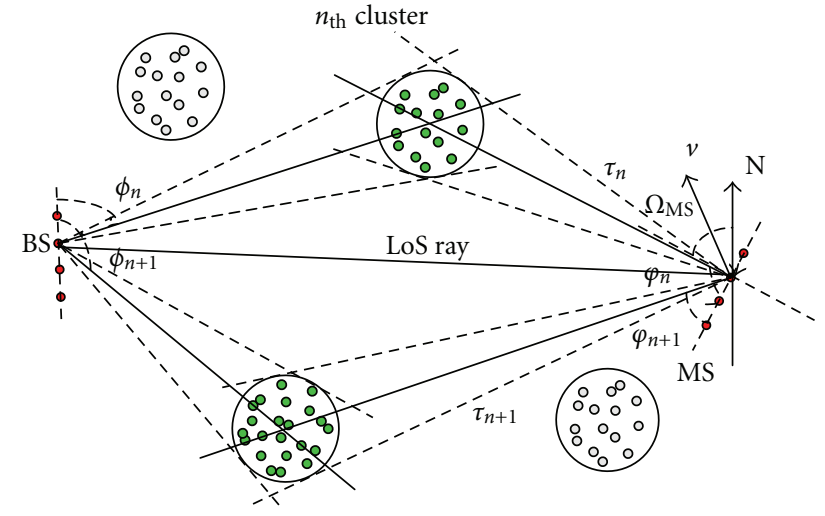

FIGURE 2: Single link in WINNER II channel model.

(1) Generate the Delays $\tau$. Delays are drawn randomly from the delay distribution defined in [12]. With exponential delay distribution, calculate

$$
\tau_{n}^{\prime}=-r_{\tau} \sigma_{\tau} \ln \left(X_{n}\right),
$$

where $r_{\tau}$ is the delay distribution proportionality factor ( $3.8 \mathrm{~ns}$ in D2a scenario), and $\sigma_{\tau}$ is delay spread defined as 40 ns. $X_{n} \sim \operatorname{Uni}(0,1)$ and cluster index $n=1, \ldots, 8$.

Normalize the delays by subtracting with minimum delay and sort the normalized delays to descending order as follows:

$$
\tau_{n}=\operatorname{sort}\left(\tau_{n}^{\prime}-\min \left(\tau_{n}^{\prime}\right)\right) .
$$

In the case of LoS condition, additional scaling of delays is required to compensate the effect of LoS peak addition to the delay spread as follows:

$$
\tau_{n}^{\mathrm{LoS}}=\tau_{n} / D
$$

where $D=0.7705-0.0433 K+0.0002 K^{2}+$ $0.000017 K^{3} ; K[\mathrm{~dB}]$ is the Ricean $K$-factor.

(2) Generate the Cluster Powers P. With exponential delay distribution the cluster powers are determined by

$$
P_{n}^{\prime}=\exp \left(-\tau_{n} \frac{r_{\tau}-1}{r_{\tau} \sigma_{\tau}}\right) \cdot 10^{-Z_{n} / 10},
$$

where $Z_{n} \sim N(0, \zeta)$ is the per cluster shadowing term in $[\mathrm{dB}]$. Average the power so that sum power of all clusters is equal to one as

$$
P_{n}=\frac{P_{n}^{\prime}}{\sum_{n=1}^{N} P_{n}^{\prime}} .
$$

Assign the power of each ray within a cluster as $P_{n} / M$, where $M=20$ is the number of rays per cluster.

For the two strongest clusters, say $n=1$ and 2, rays are spread in delay to three subclusters (per cluster), and twenty rays of a cluster are mapped to sub-clusters as presented in Table 1. 
TABLE 1: Subcluster information for intracluster delay spread clusters.

\begin{tabular}{lccc}
\hline Sub-cluster & Mapping to rays & Power & Delay offset \\
\hline 1 & $1,2,3,4,5,6,7,8,19,20$ & $10 / 20$ & $0 \mathrm{~ns}$ \\
2 & $9,10,11,12,17,18$ & $6 / 20$ & $5 \mathrm{~ns}$ \\
3 & $13,14,15,16$ & $4 / 20$ & $10 \mathrm{~ns}$ \\
\hline
\end{tabular}

TABLE 2: Ray offset angles within a cluster, given for $1^{\circ} \mathrm{rms}$ angle spread.

\begin{tabular}{lc}
\hline Ray number $m$ & Basis vector of offset angles $\alpha_{m}$ \\
\hline 1,2 & 0.0447 \\
3,4 & 0.1413 \\
5,6 & 0.2492 \\
7,8 & 0.3715 \\
9,10 & 0.5129 \\
11,12 & 0.6797 \\
13,14 & 0.8844 \\
15,16 & 1.1481 \\
17,18 & 1.5195 \\
19,20 & 2.1551 \\
\hline
\end{tabular}

(3) Generate the Azimuth Arrival Angles $\varphi$ and Azimuth Departure Angles $\phi$. The AOA for the $n$th cluster is

$$
\varphi_{n}=\left(X_{n} \varphi_{n}^{\prime}+Y_{n}\right)-\left(X_{n} \varphi_{1}^{\prime}+Y_{1}\right)+\varphi_{\mathrm{LOS}},
$$

where

$$
\begin{gathered}
\varphi_{n}^{\prime}=\frac{2 \sigma_{\mathrm{AOA}} \sqrt{-\ln \left(P_{n} / \max \left(P_{N}\right)\right)}}{C_{\mathrm{LOS}}}, \\
\sigma_{\mathrm{AOA}}=\sigma_{\varphi / 1.4}, \\
C_{\mathrm{LOS}}=C \times\left(1.1035-0.028 K-0.002 K^{2}+0.00001 K^{3}\right) .
\end{gathered}
$$

In the above equation $\sigma_{\mathrm{AOA}}$ is the standard deviation of arrival angles (factor 1.4 is the ratio of Gaussian std and corresponding RMS spread). $\varphi_{\mathrm{LOS}}$ is the LoS direction and component $Y_{n} \sim N\left(0, \sigma_{\mathrm{AOA}} / 5\right)$. Add the offset angles from Table 2 to cluster angles as

$$
\varphi_{n, m}=\varphi_{n}+c_{\mathrm{AOA}} \alpha_{m},
$$

where $\varphi_{n, m}$ is the AOA for each ray $m$ of each cluster $n$; $c_{\mathrm{AOA}}$ is the cluster-wise rms azimuth spread of AOA $\left(c_{\mathrm{AOA}}=\right.$ $3^{\circ}$ in D2a scenarios). The corresponding offset angle $\alpha_{m}$ is taken from Table 2. For departure angle $\phi_{n}$, the procedure is analogous.

(4) Draw the Random Initial Phase. $\left\{\Phi_{n, m}^{V V}, \Phi_{n, m}^{V H}, \Phi_{n, m}^{H V}, \Phi_{n, m}^{H H}\right\}$ are the random initial phases for each ray $m$ of each cluster $n$ and for four different polarization combinations $(v v, v h, h v, h h)$. Distribution for the initial phases is uniform, Uni $(-\pi, \pi)$. In the LoS case, draw also random initial phases $\left\{\Phi_{\mathrm{LoS}}^{V V}, \Phi_{\mathrm{LoS}}^{H H}\right\}$ for both $V V$ and $H H$ polarisations.
(5) Generate the Cross-Polarisation Power Ratios (XPR) $\kappa_{n, m}$ for Each Ray $m$ of Each Cluster n. XPR is log-normal distributed. Draw XPR values as

$$
\kappa_{n, m}=10^{X / 10},
$$

where $X \sim N(\sigma, \mu)$ is Gaussian distributed with $\sigma$ and $\mu$ from [12] for XPR.

(6) Generate the Channel Coefficients for Each Cluster $n$ and Each Receiver and Transmitter Element Pair $u, s$. For the clusters in D2a, say $n=1,2, \ldots, 8$, and uniform linear arrays (ULA), the channel coefficients are given by

$$
H_{u, s, n}(t)=\sqrt{\frac{1}{K_{R}+1}} H_{u, s, n}^{\prime}(t)+\delta(n-1) \sqrt{\frac{K_{R}}{K_{R}+1}} H_{0}(t),
$$

where $\delta(\cdot)$ is the Dirac's delta function and $K_{R}$ is the Ricean $K$-factor converted to linear scale. $H_{0}(t)$ is the channel coefficient corresponding to the single LoS ray. $H_{u, s, n}^{\prime}(t)$ is the non-LoS channel coefficient component. $H_{0}(t)$ and $H_{u, s, n}^{\prime}(t)$ are given by

$$
\begin{aligned}
& H_{0}(t)=\left[\begin{array}{c}
F_{t x, s, V}\left(\phi_{\mathrm{LoS}}\right) \\
F_{t x, s, H}\left(\phi_{\mathrm{LoS}}\right)
\end{array}\right]^{T} \\
& \cdot\left[\begin{array}{cc}
\exp \left(j \Phi_{\mathrm{LoS}}^{V V}\right) & 0 \\
0 & \exp \left(j \Phi_{\mathrm{LoS}}^{H H}\right)
\end{array}\right]\left[\begin{array}{l}
F_{r x, u, V}\left(\varphi_{\mathrm{LoS}}\right) \\
F_{r x, u, H}\left(\varphi_{\mathrm{LoS}}\right)
\end{array}\right] \\
& \cdot \exp \left(j d_{s} 2 \pi \lambda_{0}^{-1} \sin \left(\phi_{\mathrm{LoS}}\right)\right) \\
& \cdot \exp \left(j d_{u} 2 \pi \lambda_{0}^{-1} \sin \left(\varphi_{\mathrm{LoS}}\right)\right) \cdot \exp \left(j 2 \pi \nu_{\mathrm{LoS}} t\right), \\
& H_{u, s, n}^{\prime}(t)=\sqrt{P_{n}} \sum_{m=1}^{M}\left[\begin{array}{c}
F_{t x, s, V}\left(\phi_{n, m}\right) \\
F_{t x, s, H}\left(\phi_{n, m}\right)
\end{array}\right]^{T} \\
& \cdot\left[\begin{array}{cc}
\exp \left(j \Phi_{n, m}^{V V}\right) & \sqrt{\kappa_{n, m}} \exp \left(j \Phi_{n, m}^{V H}\right) \\
\sqrt{\kappa_{n, m}} \exp \left(j \Phi_{n, m}^{H V}\right) & \exp \left(j \Phi_{n, m}^{H H}\right)
\end{array}\right] \\
& \cdot\left[\begin{array}{l}
F_{r x, u, V}\left(\varphi_{n, m}\right) \\
F_{r x, u, H}\left(\varphi_{n, m}\right)
\end{array}\right] \cdot \exp \left(j d_{s} 2 \pi \lambda_{0}^{-1} \sin \left(\phi_{n, m}\right)\right) \\
& \text { - } \exp \left(j d_{u} 2 \pi \lambda_{0}^{-1} \sin \left(\varphi_{n, m}\right)\right) \cdot \exp \left(j 2 \pi v_{n, m} t\right) \text {, }
\end{aligned}
$$

where $F_{r x, u, V}$ and $F_{r x, u, H}$ are the receiving antenna element $u$ field patterns for vertical and horizontal polarizations, respectively, similarly $F_{t x}$ is the transmitting antenna patterns. $d_{s}$ and $d_{u}$ are antenna spacing $(\mathrm{m})$ between transmitter elements and receiver elements, respectively, and $\lambda_{0}$ is the wavelength on carrier frequency. $\nu_{\text {LoS }}$ and $\nu_{n, m}$ are the Doppler frequency shifts for the LoS ray and each ray $m$ of each cluster $n$, respectively. The Doppler frequency 
component is calculated from AOA, MS speed $v$, and direction of travel $\theta_{v}$ as follows:

$$
\begin{gathered}
\nu_{\mathrm{LoS}}=\frac{\|v\| \cos \left(\varphi_{\mathrm{LoS}}-\theta_{v}\right)}{\lambda_{0}}, \\
\nu_{n, m}=\frac{\|v\| \cos \left(\varphi_{n, m}-\theta_{v}\right)}{\lambda_{0}} .
\end{gathered}
$$

The CIR for each receiver and transmitter element pair $u, s$ is given by

$$
H_{(u, s)}(t)=\sum_{n=1}^{N_{c}} H_{u, s, n}\left(t-\tau_{n}\right)
$$

2.3. Path Loss Model. Path loss model for the WINNER II D2a scenario has been developed based on results of measurements carried out within WINNER, and it is formed as

$$
\mathrm{PL}=21.5 \log _{10}(D)+44.2+20 \log _{10}\left(\frac{f_{c}}{5.0}\right)
$$

where $D$ is the distance between the transmitter and the receiver in $(\mathrm{m}), f_{c}$ is the system frequency in $(\mathrm{GHz})$. Path loss factor PL is given as a parameter, which is multiplied to channel matrices.

\section{Maximum LoS MIMO Capacity Criterion}

Using the simplified maximum capacity criterion in LoS MIMO systems, the problem of reduced capacity in a LoS scenario can be overcome [8]. The validity of the new capacity criterion implementation was investigated by WINNER II channel model in the next section.

3.1. MIMO Channel Capacity. To assess the performance of the stochastic channel in the presence of scatter, the notion of ergodic capacity must be employed. Note that the transmit power is equal to $P_{T} / S$ (at all transmit elements) as UPA (uniform power allocation) is used. The ergodic capacity of a MIMO system to be given by [13]

$$
C=E\left\{\log _{2}\left(\operatorname{det}\left(I_{U}+\frac{\rho}{S} H H^{H}\right)\right)\right\},
$$

where $\rho$ corresponds to the average received signal-to-noise ratio (SNR) at the input of the receiver, and $[\cdot]^{H}$ denotes the conjugate transpose. Extremely, the capacity in (15) is maximized for $H H^{H}=S I_{U}$, and this response corresponds to a channel with perfectly orthogonal MIMO subchannels.

3.2. Maximum $U \times S$ Capacity Criterion. It has been demonstrated that the capacity in LoS MIMO channel (ignoring any scatter components at this stage) is maximized, when the following criterion is fulfilled [8]:

$$
d_{s} d_{u}=\lambda\left(\frac{1}{S}+p\right) \frac{D}{\sin \omega \sin \theta}, \quad \forall p \in \mathbb{Z}
$$

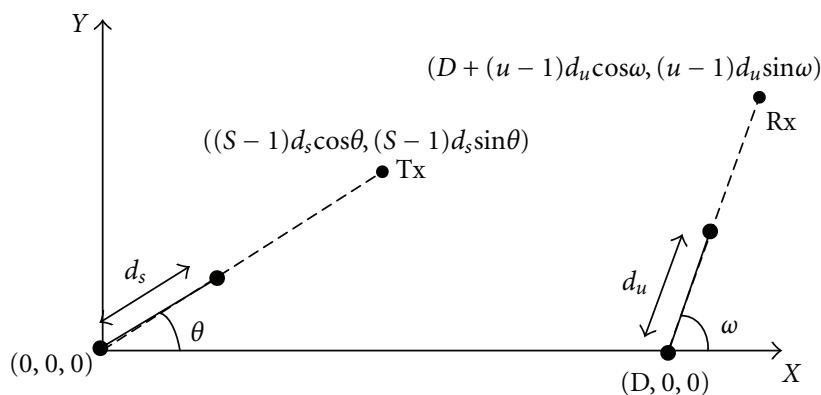

Figure 3: Positioning of the elements in a $U \times S$ MIMO system.

where $D$ is the Tx-Rx distance, and [8] defines the distance between the first element of each array to equal $D$. The $3 \mathrm{D}$ geometric configuration is shown in Figure 3. $\theta$ and $\omega$ are the orientations of the arrays for Tx and Rx, respectively. Thus, by knowing the carrier frequency and the Tx-Rx distance, the optimal spacing can be easily calculated from the maximum LoS MIMO capacity criterion. In other words, this criterion for perfectly orthogonal MIMO subchannels architectures defines a number of MIMO architectures with antenna arrays fixed at optimal points.

\section{Simulation Results}

The high speed railway scenarios have its distinctive characteristics, such as a relatively long distance between transmitters and receivers, the high speed of $350 \mathrm{~km} / \mathrm{h}$ or above, and a number of scatters. But the maximum capacity criterion only considers the LoS component of the channel response. The characteristics of railway scenarios must be accounted for evaluating the criterion. Thus, in this section, we evaluate the criterion (16) in practical high speed railway scenarios described in Figure 1.

We explore a $2 \times 2$ MIMO system operating at $2.6 \mathrm{GHz}$. And the orientations of the antenna arrays for $\mathrm{Tx}$ and $\mathrm{Rx}$ are perpendicular to the track as $\theta=90^{\circ}$ and $\omega=$ $90^{\circ}$, respectively. Then, the maximum capacity criterion is simplified as $d_{s} d_{u}=\lambda D / 2, p=0$. Assuming interelement spacing $d_{s}$ for reconfigurable antenna arrays equal to $1 \mathrm{~m}$, the $d_{u}$ can be calculated. In the high speed railway scenarios, the separated distance $D$ between BS and MS is usually in order of hundreds of meters and is much larger than $d_{\mathrm{BS}}$, therefore the approximation as $D=\sqrt{l^{2}+d_{\mathrm{BS}}^{2}} \approx l$ is used for simplification, where $l$ is the separated distance between the projection of the BS and the projection of the train on $X$-axis. In Figure 4, we have optimal interelement spacings $d_{s}=1 \mathrm{~m}$, and $d_{u}=28.85 \mathrm{~m}$ for the point $x=0(l=500 \mathrm{~m})$. The $K$-factor is set to $9 \mathrm{~dB}$. The ergodic capacity is plotted as a function of SNR for different interelement spacings. The simulated curves are generated according to (15).

It can be easily seen that the ULA with optimal antenna spacings shows superiority to other geometries over the entire SNR range. When the SNR reaches $20 \mathrm{~dB}$, the capacity of optimal case is about $5 \mathrm{bps} / \mathrm{Hz}$ higher than that of ULA with $10 \lambda$ interelement spacings. In addition, the ULA with $10 \lambda$ interelement spacings shows superiority to the one with 


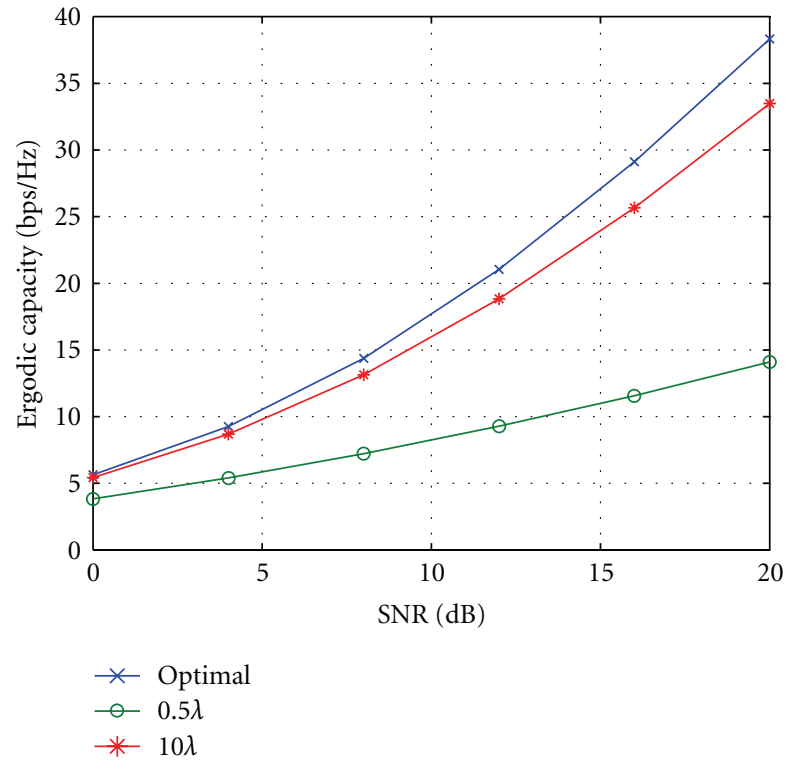

FIGURE 4: Ergodic capacity as a function of SNR for different antenna spacings ( $K$-factor $=9 \mathrm{~dB}, l=275 \mathrm{~m})$.

$0.5 \lambda$ interelement spacings. The reason is that placing the antenna elements far apart results in high resolution and low correlation of channel matrix and then it gives the high capacity.

4.1. Displacement. The criterion for high-rank LoS MIMO channels defines a number of MIMO architectures for systems with ULAs fixed at optimal locations. However, in high speed railway situations, there is a need for high capacity over an area, rather than to a fixed point. To examine the sensitivity of the performance of maximum capacity architectures in high speed railways, the capacity is now evaluated as a function of the displacement from the optimal point. The simulation is explored in the location range from 0 to $500 \mathrm{~m}$, which is the whole trajectory, that is from the midpoint between two BSTs up to the point closest to another BST. And it is equivalent to a $500 \mathrm{~m}$ displacement range refering to the optimal point $x=0$. The optimal interelement spacings are $d_{s}=1 \mathrm{~m}$ and $d_{u}=28.85 \mathrm{~m}$. The received power gradually changes.

The variation of capacity with different displacements is shown in Figure 5. From this figure, the performance of optimal case shows superiority to other geometries over the entire trajectory. Above a displacement of $350 \mathrm{~m}$ or so, the curve of capacity is escalating faster and faster. The reason is the increased received power and the decreased degree of scattering. When the displacement reaches $500 \mathrm{~m}$, the capacity of optimal case is about $1.5 \mathrm{bps} / \mathrm{Hz}$ higher than that of ULA with $0.5 \lambda$ interelement spacings. In addition, the ULA with $10 \lambda$ interelement spacings shows superiority to the one with $0.5 \lambda$ interelement spacings. The reason is the high resolution and the low correlation of channel matrix.

The sensitivity of the capacity performance for the optimum case is investigated by means of a narrow distance window, see Figure 6. The simulation is explored in the range

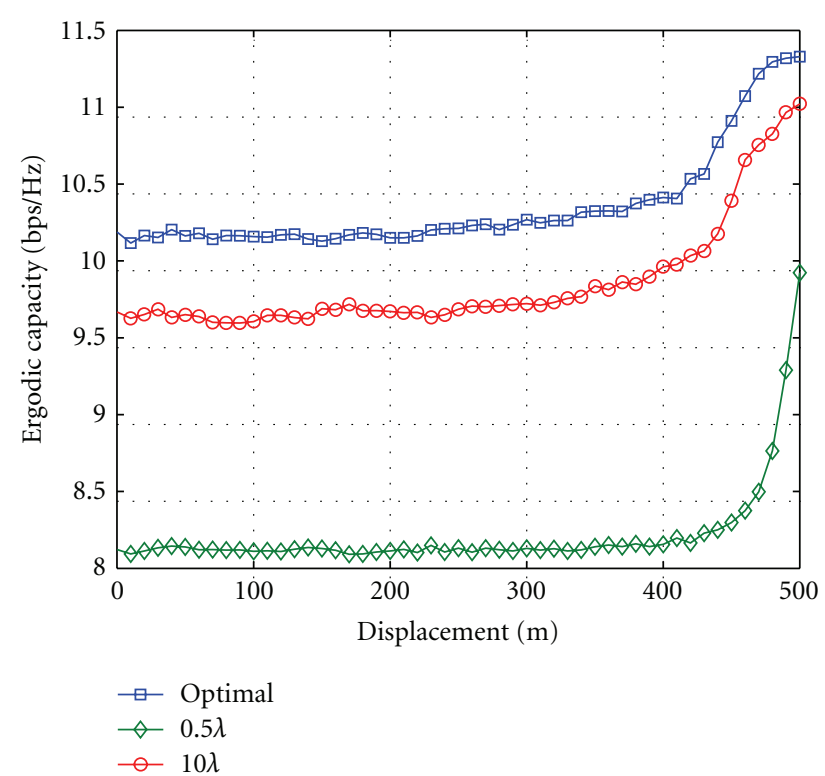

FIGURE 5: Ergodic capacity as a function of the displacement from the optimum point.

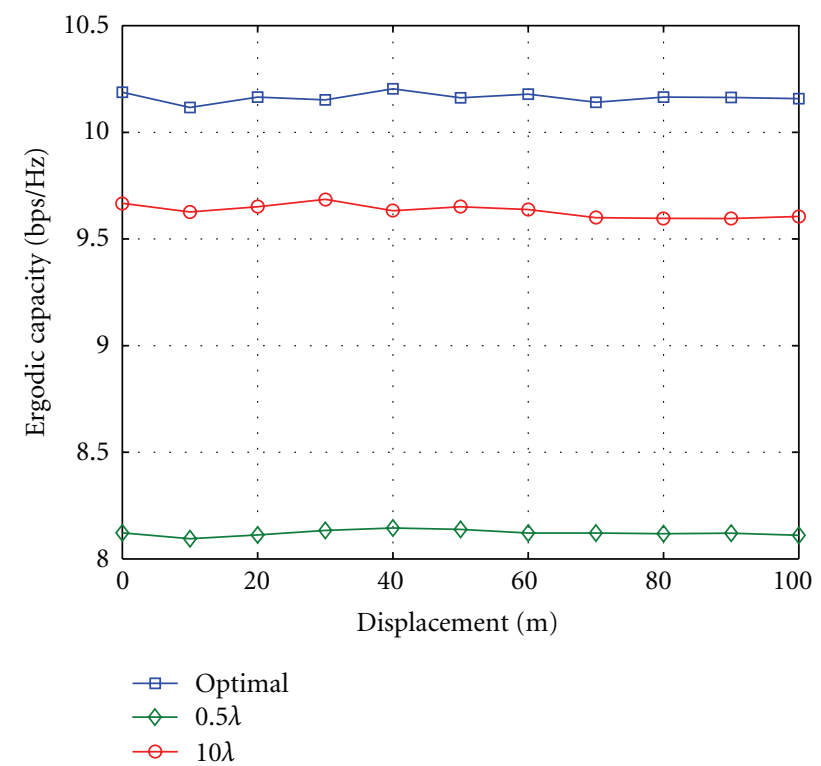

FIGURE 6: Ergodic capacity as a function of the displacement from the optimum point.

of $0 \mathrm{~m}$ to $100 \mathrm{~m}$, which is equivalent to a $100 \mathrm{~m}$ displacement range, refer to the optimal point $x=0 \mathrm{~m}$. From this figure, it is clear that the capacity is relatively insensitive to small displacements from the optimum point. The reason is that the angle changes of $\theta$ and $\omega$ are very small due to short displacements. According to (16), the optimal interelement spacing is local optimum for the premise of $\sin (\theta)$ and $\sin (\omega)$ is basically invariable. So, the same reconfiguration antenna array used in a small regional is feasible.

4.2. ULA Azimuthal Orientation. There are some disadvantages for antenna array orientation in railway scenarios due 


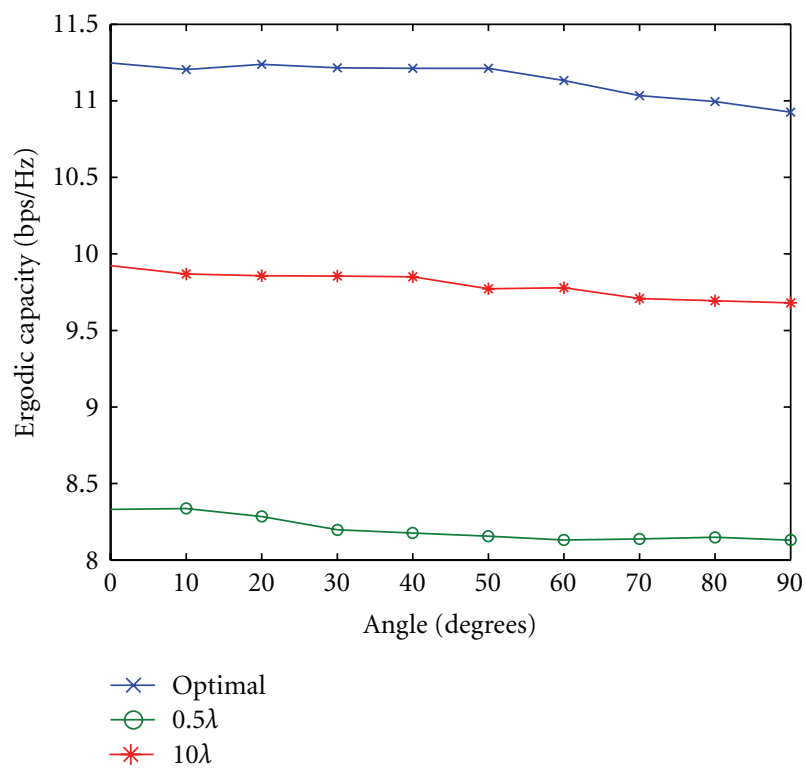

FIGURE 7: Ergodic capacity as a function of the angle deviation from the optimum angle $(\rho=20 \mathrm{~dB})$.

to the fast moving of the train and the rapid change of the radio propagation environments. It is difficult to deal with this fast fading. On the other hand, it has some advantages that the route of the train is fixed and the path can be predicted. So, some special antenna types or programs can be chosen. It is clear that there is a dependence of the capacity to the azimuthal orientation of the two arrays (angles $\theta$ and $\omega$ ) from the maximum capacity criterion of (16). This dependence is now examined using the same channel model as before.

The effect of ULA azimuthal orientation at MS ends on the capacity is shown in Figure 7. The units of angle in MS coordinate axes are degree. The ergodic channel capacity is obtained at $\rho=20 \mathrm{~dB}$. Each point in Figure 7 stands for different MS azimuthal orientation and fixed BS azimuthal orientation at the optimal point $x=0$. At each process of simulation, the antenna orientations of BS and the train are fixed. The results show a little sensitivity (in terms of capacity) to the orientation of the MS arrays. In detail, the channel capacity is seen to vary between the minimum $(C \min =10.93 \mathrm{bps} / \mathrm{Hz})$ and the maximum $(C \max =$ $11.25 \mathrm{bps} / \mathrm{Hz}$ ) values about the curve for the optimal one. The results suggested that this sensitivity needs not focus too much on the design of any practical MIMO system.

(1) Maximizing Capacity in Viaduct Scenarios Using Reconfigurable Antenna Arrays. Now theory and methods for exploiting the potential of reconfigurable RF front-ends in high speed railway are not fully developed. The results of simulation offer a reference for reconfigurable antenna arrays on maximizing the capacity of MIMO wireless communication links in high speed railway scenarios. And there is measure data based on Propsound measurements, which is operated in viaduct scenarios [11]. Then, we put forward two proposals through a combination of simulation results and the measurement data.
TABLE 3: The parameters for optimum proposals.

\begin{tabular}{lcccc}
\hline & RA & TA & CA & AA \\
\hline The region & $(0,230)$ & $(230,320)$ & $(320,480)$ & $(480,500)$ \\
$(X$ axis, $m)$ & & & & \\
$D$ for proposal 1 $(m)$ & 385 & 225 & 100 & 14 \\
$D$ for proposal 2 $(m)$ & 500 & 500 & 500 & 500 \\
$d_{u}$ for proposal 1 $(m)$ & 22.23 & 12.96 & 5.76 & 0.81 \\
$d_{u}$ for proposal 2 $(m)$ & 28.85 & 28.85 & 28.85 & 28.85 \\
\hline
\end{tabular}

According to [11], the coverage area of the base station is divided into 4 regions as remote area (RA), toward area (TA), close area (CA), and arrival area (AA). We can design different regions in the area that are involved in different reconfigurable antenna arrays because of the rapidly change of separate distances and different small-scale fading characteristics. The maximum capacity criterion is introduced in the previous section, and then two practical optimum proposals are afforded.

Optimum Proposal 1. Select four midpoints of the four linear regions as the optimal locations and use them to calculate optimal interelement spacings for the different reconfiguration antenna arrays involved in the four regions. The interelement spacings of ULA are adjusted for different regions, when the train travels through the coverage area of base stations.

The maximum capacity criterion defines a number of MIMO architectures for systems with antenna arrays fixed at optimal locations. However, there is a need for high capacity over an area, rather than to a fixed point. In the simulations, to examine the sensitivity of the performance of maximum capacity architectures under high speed railway scenarios, we can determine the advantage of the optimal antenna array basically stable in the small displacements range.

Optimum Proposal 2. Select the edge point of base station coverage as the optimal location and obtain the optimal interelement spacing of the reconfigurable antenna array involved in all regions. The interelement spacing of ULA maintains invariable when the train travels through the linear coverage. This proposal maximizes the capacity of the coverage edge and minimizes the correlation of channel matrix in the coverage.

The corresponding parameters for optimum proposals are taken from Table 3 .

4.3. Scattering. In the maximum LoS MIMO capacity criterion, only LoS component of the channel response is considered. In railway scenarios, some degrees of scatterings are always present in the radio channel, and, hence, their effects must be accounted for the design of the MIMO system.

In Figure 8, the ergodic capacity is plotted against the Ricean $K$-factor and compared with two reference geometries. It demonstrates again the superiority of the optimal design obtained by maximum capacity criterion. 


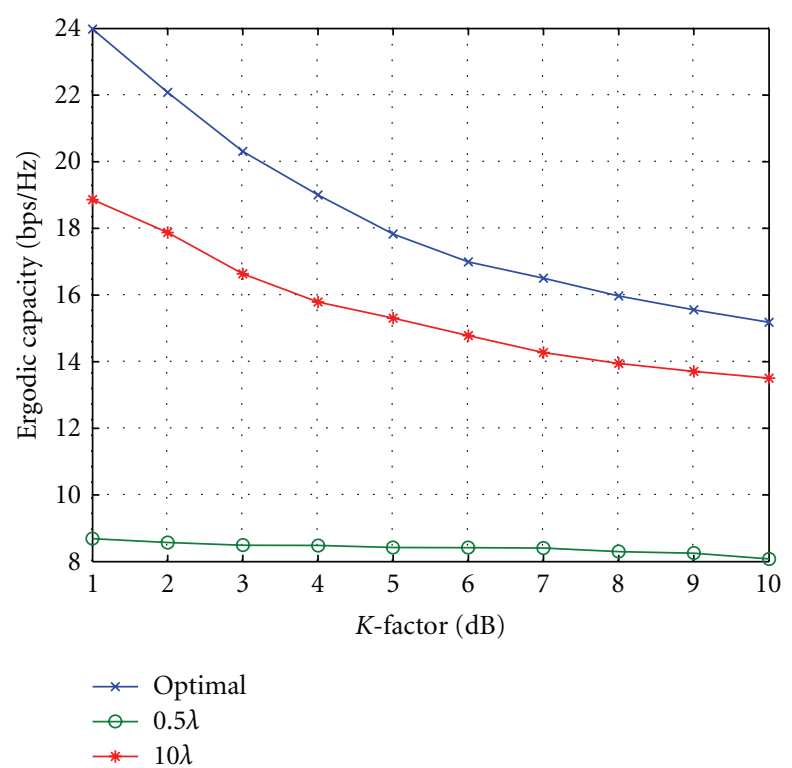

FIgURe 8: Ergodic capacity as a function of $K$-factor for different antenna spacings $(\rho=10 \mathrm{~dB}, x=500 \mathrm{~m})$.

And meanwhile we see the capacities for ULAs with different antenna spacings decrease when $K$-factor increases. The optimal design is not expected in this result because the curve for optimal interelement spacings should increase with the increment of $K$-factor, and the reason is high-rank channel for the optimal case [8]. And the unsatisfactory result can be attributed to the alterations of eigenvalue profiles, which are not full rank when $K$-factor changes. Meanwhile, UPA used in our investigation becomes quite suboptimal since an amount of power is wasted inevitably on the vanishing or relatively weak spatial dimensions.

\section{Conclusion}

In this paper, we study the validity of the maximum capacity criterion in real high speed railway environment, which is applied to realize high-rank LoS MIMO channels. D2a scenario of WINNER II channel model is adopted and it is a realistic high speed railway transmission multipath propagation channel. The ergodic capacity is used as the index to discuss the performance of reconfigurable antenna arrays. Numerical results demonstrate that significant capacity gains are achievable by simply adjusting antenna spacing according to the maximum capacity criterion. And we obtain relatively low sensitivity of the optimal antenna arrays to displacement from the optimal point and angle. So, we put forward two proposals for reconfigurable antenna arrays so as to maximize LoS MIMO capacity in the high speed railway scenarios. Then we find that the antenna array geometries obtained from the criterion are suboptimal, since the ergodic capacity decreases with the increment of $K$-factor. Thus, it is very necessary to carry on a more thorough research on this optimal methodology for constructing high-rank LoS MIMO channels in high speed railway communication system.

\section{Acknowledgments}

The authors would like to express their great thanks to the support from the National Natural Science Foundation of China under Grant 61222105, Beijing Municipal Natural Science Foundation under Grant 4112048, the Fundamental Research Funds for the Central Universities under Grant 2010JBZ008, Program for New Century Excellent Talents in University under Grant NCET-09-0206, and the Key Project of State Key Laboratory under Grant RCS2011ZZ008.

\section{References}

[1] Y. Li and L. J. Cimini Jr., "Bounds on the interchannel interference of OFDM in time-varying impairments," IEEE Transactions on Communications, vol. 49, no. 3, pp. 401-404, 2001.

[2] A. Grant, "Rayleigh fading multi-antenna channels," Eurasip Journal on Applied Signal Processing, vol. 2002, no. 3, pp. 316329, 2002.

[3] M. Kang and M. S. Alouini, "Capacity of MIMO Rician channels," IEEE Transactions on Wireless Communications, vol. 5, no. 1, pp. 112-122, 2006.

[4] S. Jin, X. Gao, and X. You, "On the ergodic capacity of rank-1 Ricean-fading MIMO channels," IEEE Transactions on Information Theory, vol. 53, no. 2, pp. 502-517, 2007.

[5] F. Bøhagen, P. Orten, and G. E. Øien, "Construction and capacity analysis of high-rank line-of-sight MIMO channels," in Proceedings of the IEEE Wireless Communications and Networking Conference (WCNC '05), pp. 432-437, March 2005.

[6] F. Bøhagen, P. Orten, and G. E. Øien, "Design of optimal high-rank line-of-sight MIMO channels," IEEE Transactions on Wireless Communications, vol. 6, no. 4, pp. 1420-1424, 2007.

[7] I. Sarris and A. Nix, "Design and performance assessment of maximum capacity MIMO architectures in line-of-sight," IEE Proceedings on Communications, vol. 153, no. 4, pp. 482-488, 2006.

[8] I. Sarris and A. R. Nix, "Design and performance assessment of high-capacity MIMO architectures in the presence of a line-ofsight component," IEEE Transactions on Vehicular Technology, vol. 56, no. 4, pp. 2194-2202, 2007.

[9] M. Matthaiou, D. I. Laurenson, and C. X. Wang, "Capacity study of vehicle-to-roadside MIMO channels with a Line-ofSight component," in Proceedings of the International ICST Conference on Communications and Networking in China (CHINACOM '10), pp. 1-5, 2010.

[10] L. Gao, Z. Zhong, B. Ai, and L. Xiong, "Estimation of the Ricean $\mathrm{K}$ factor in the high speed railway scenarios," in Proceedings of the IEEE Wireless Communications and Networking Confenrence (WCNC'08), pp. 775-779, 2008.

[11] L. Liu, T. Cheng, Q. Jia-Hui, and C. Hou-Jin, "Research on small-scale fading characteristics of wireless channels in rural aera of high-speed railway," Journal of the China Railway Society, vol. 5, pp. 41-46, 2012.

[12] P. Kyosti et al., "WINNER II channel models, IST-WINNER D1.1.2," vol. 11, 2007.

[13] D. Tse and P. Vishwanath, Fundamentals of Wireless Communications, Cambridge University Press, Cambridge, UK, 2005. 

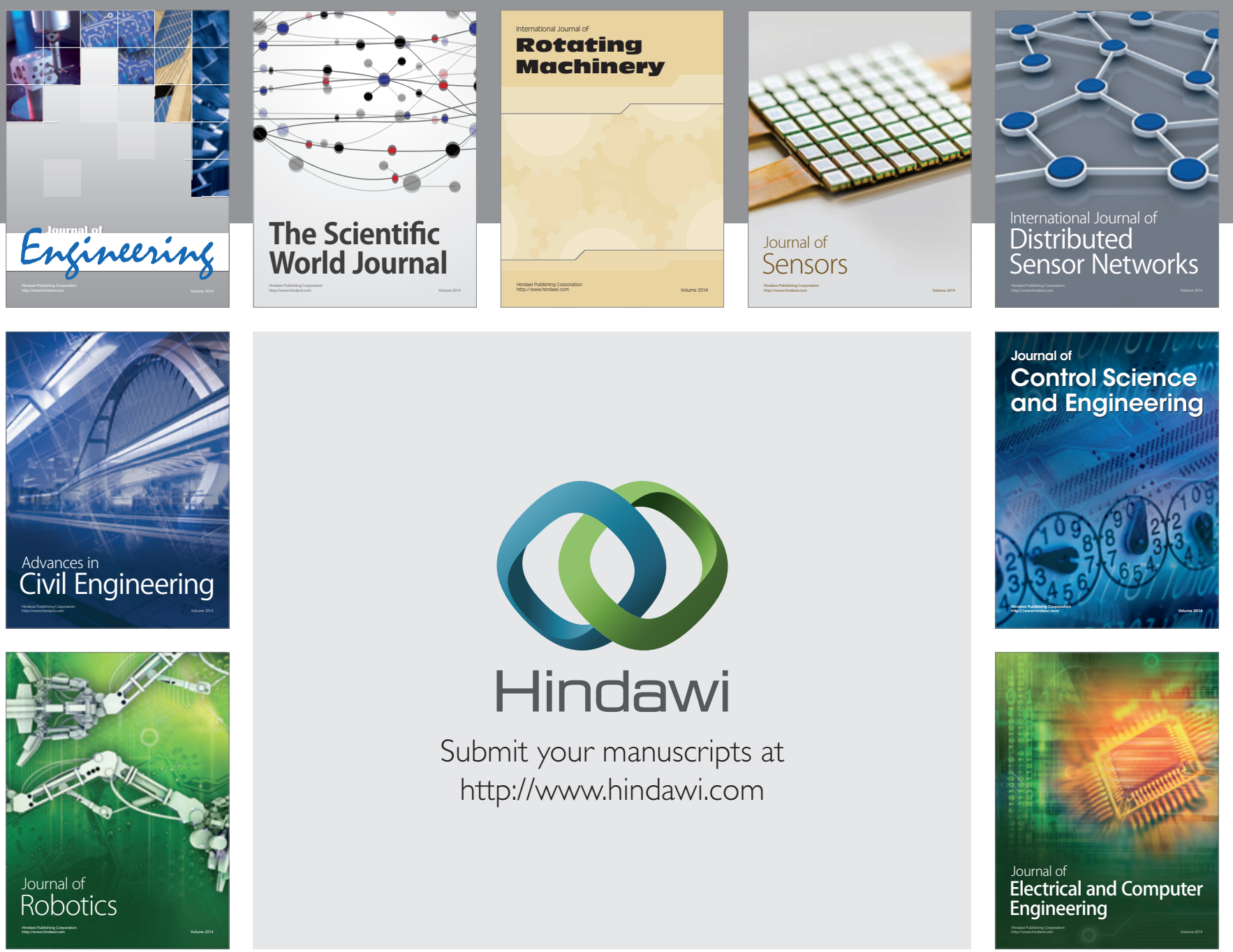

Submit your manuscripts at

http://www.hindawi.com
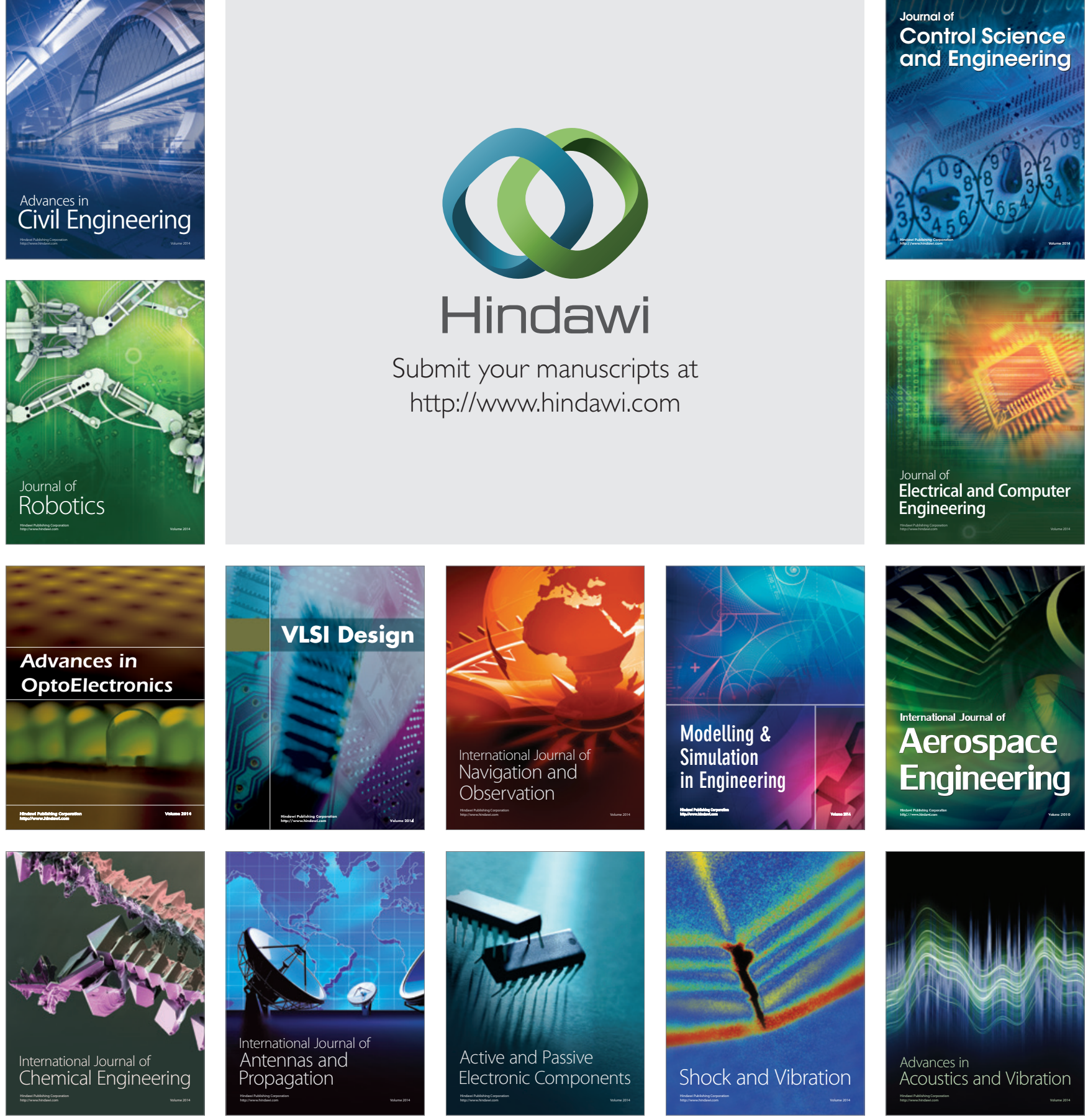